\title{
Toxicological Profile of Biological Environment of Two Elastodontic Devices
}

\author{
Stefania Dinu 1,2,+(D), Roxana Buzatu ${ }^{3,+}(\mathbb{D}$, Ioana Macasoi 4,5,*, Malina Popa 1,2,*, Cristian Sebastian Vlad 6 , \\ Iasmina Marcovici ${ }^{4,5}$, Iulia Pinzaru 4,5 ${ }^{(D}$, Cristina Adriana Dehelean ${ }^{4,5}$, Elena-Alina Moacă 4,5 , \\ Lucian Barbu-Tudoran 7,8 (D) and Marius Pricop 9
}

1 Department of Pedodontics, Faculty of Dental Medicine, Victor Babeş University of Medicine and Pharmacy Timisoara, 9 No., Revolutiei Bv., 300041 Timişoara, Romania; dinu.stefania@umft.ro

2 Pediatric Dentistry Research Center, Faculty of Dental Medicine, Victor Babeş University of Medicine and Pharmacy Timisoara, 9 No., Revolutiei Bv., 300041 Timişoara, Romania

3 Department of Dental Aesthetics, Faculty of Dental Medicine, Victor Babeş University of Medicine and Pharmacy Timisoara, 9 No., Revolutiei Bv., 300041 Timişoara, Romania; roxana.buzatu@umft.ro

4 Research Center for Pharmaco-Toxicological Evaluations, Faculty of Pharmacy, Victor Babeş University of Medicine and Pharmacy Timisoara, Eftimie Murgu Square No. 2, 300041 Timişoara, Romania; iasmina.marcovici@umft.ro (I.M.); iuliapinzaru@umft.ro (I.P.); cadehelean@umft.ro (C.A.D.); alina.moaca@umft.ro (E.-A.M.)

5 Departament of Toxicology and Drug Industry, Faculty of Pharmacy, Victor Babeş University of Medicine and Pharmacy Timisoara, 2nd Eftimie Murgu Sq., 300041 Timişoara, Romania

6 Department of Pharmacology, Faculty of Medicine, Victor Babeş University of Medicine and Pharmacy Timisoara, Eftimie Murgu Square No. 2, 300041 Timişoara, Romania; vlad.cristian@umft.ro

check for updates

Citation: Dinu, S.; Buzatu, R.; Macasoi, I.; Popa, M.; Vlad, C.S.; Marcovici, I.; Pinzaru, I.; Dehelean, C.A.; Moacă, E.-A.; Barbu-Tudoran, L.; et al. Toxicological Profile of Biological Environment of Two Elastodontic Devices. Processes 2021, 9, 2116. https://doi.org/10.3390/ pr9122116

Academic Editor: Tao Sun

Received: 16 October 2021

Accepted: 19 November 2021

Published: 24 November 2021

Publisher's Note: MDPI stays neutral with regard to jurisdictional claims in published maps and institutional affiliations.

Copyright: (c) 2021 by the authors. Licensee MDPI, Basel, Switzerland. This article is an open access article distributed under the terms and conditions of the Creative Commons Attribution (CC BY) license (https:// creativecommons.org/licenses/by/ $4.0 /)$.
7 Electron Microscopy Laboratory "Prof. C. Craciun", Faculty of Biology \& Geology, "Babes-Bolyai" University, 5-7 Clinicilor Street, 400006 Cluj-Napoca, Romania; lucian.barbu@ubbcluj.ro

8 Electron Microscopy Integrated Laboratory, National Institute for R\&D of Isotopic and Molecular Technologies, 67-103 Donat Street, 400293 Cluj-Napoca, Romania

9 Department of Maxillofacial Surgery, Faculty of Dental Medicine, Victor Babeş University of Medicine and Pharmacy Timisoara, 9 No., Revolutiei Bv., 300041 Timişoara, Romania; pricop.marius@umft.ro

* Correspondence: macasoi.ioana@umft.ro (I.M.); popa.malina@umft.ro (M.P.)

+ Authors with equal contribution.

Abstract: Malocclusion and teething problems are common health problems globally, affecting people of all ages, especially children and adolescents. In addition to the pathophysiological complications associated with orthodontic problems, they also affect the well-being of the individual. Orthodontic appliances are frequently used, even from an early age, and their activity in different biological environments is very varied and incompletely described. Due to these considerations, the purpose of the study was to evaluate the toxicological profile of the biological environment (saliva at three $\mathrm{pH}$ values: 3, 7, and 10) of two elastodontic orthodontic appliances: Myobrace (MB) and LM TrainerTM 2 (LMD). In vitro techniques applied were conducted on human keratinocytes to evaluate cell viability (Alamar blue assay) and gene expression real-time reverse transcription-polymerase chain reaction (RT-PCR technique). In addition, it was assessed the irritating effect on the vascular plexus using as a biological model the chorioallantoic membrane of the hen's egg by applying the hen's eggchorioallantoic membrane (HET-CAM) method. The obtained results showed a decrease in cell viability up to $82 \%$ in the case of $\mathrm{LMD}$ at $\mathrm{pH}=3$, a slight increase in mRNA expression for the anti-apoptotic marker (Bcl-2 and Bcl-xL), and a decrease in mRNA expression for the pro-apoptotic marker (Bad), and any type of toxic change at the capillary level (irritation score being below 0.9). Based on the data obtained, it can be stated that MB and LMD biological environments, at different $\mathrm{pH}$ values, present a safe toxicological profile.

Keywords: malocclusion; keratinocytes; viability; gene expression; angiogenic effect 


\section{Introduction}

Malocclusion is an alteration of the developers, not necessarily a pathology. At the base of this medical condition are multiple factors, including genetic heredity, psychological and physical factors, trauma, or unhealthy habits. Unfortunately, patients who have defects of normal tooth developers are responsible for two or more etiological factors. However, some factors can be prevented by applying appropriate treatment that can cross malocclusion in time [1]. In terms of impact on oral health, malocclusion is considered one of the biggest dental problems, ranking third in the incidence of the disease after tooth decay and periodontal disease [2]. In addition to its impact on the physical appearance and psychological state of patients, malocclusion also has other physiological and pathological complications such as the increased risk of tooth decay, dental lesions, and various diseases of the temporomandibular joint [3]. Regarding the quality of life, it comprises several areas, including self-esteem, psychological, and social status. Oral health is one of the major factors that can influence well-being, especially among children and adolescents. Given that malocclusion mainly affects these age groups, children and adolescents may experience psychological discomfort that can affect their quality of life and physical and psychological functioning [4]. One of the methods of preventing these diseases is to start early orthodontic treatment, also known as a preventive and interceptive treatment. This therapeutic approach can be employed from childhood since the development of dentition [1]. The classification of malocclusion from the skeletal and dental point of view is important both from the point of view of diagnosis and from the point of view of the therapeutic approach [5]. In the early 1990s, Angle first made a classification of malocclusion in terms of the mesio-distal relationship of the teeth. This classification was based on the first remaining maxillary molar and includes three classes marked with Roman numerals (Class I, II, and III) [6]. Class I is the most common form of malocclusion and is characterized by a normal molar relationship but with incorrect tooth position. The main clinical manifestation of this class of malocclusion is the defective alignment of the anterior teeth, especially the mandibular teeth [7]. Class II of malocclusion has a lower incidence compared to class I (between $2 \%$ and $15 \%$ of cases of malocclusion in Europe) and, according to the latest classifications, can be divided into three subcategories depending on the position of maxillary lateral incisors with severity ranging from low to severe [8]. Class III malocclusion is considered a complicated maxillofacial pathology, characterized by a concave profile with the presence of mandibular protrusion, maxillary retrusion, or a combination thereof. Due to the little information regarding this subcategory of malocclusion, the specialists in the field face major problems both in terms of the correct diagnosis of the disease and the establishment of appropriate treatment [9]. The goal of early treatment is to improve dental and skeletal development. In addition, by introducing the treatment still in the developmental stage of the dentition, there is a decrease in the malocclusion and of the treatment time for the correction of the permanent dentition [10]. One of the recent approaches to orthodontic treatment is to determine the causative factors of malocclusion, such as artificial breastfeeding, frequent and prolonged use of the pacifier, or mouth breathing. Thus, by correcting these habits simultaneously with dental alignment by using elastodontic dental appliances, it is gaining more and more ground in orthodontic practice [11]. On the other hand, the other alternative approach to these dental diseases is represented by the use of bracket devices that have the disadvantage number, the most important being the high percentage of recurrence [12]. Two of the most elastodontic orthodontic appliances are Myobrace for Kids from Myofunctional Research Co. (Helensvale, Australia) (MB) and LM TrainerTM 2 (LMD). These are detachable devices composed of soft elastomeric material and aim to correct class II malocclusion. They have the advantage of acting through two major mechanisms, namely: (i) guiding the growth of teeth and (ii) correcting the mandibular posture [13]. The Myobrace device is a three-phase device designed to correct harmful habits as well as to treat jaw development problems. Among the actions it exerts at the level of the oral cavities are the correct positioning of the tongue in the upper jaw, the improvement of the oral muscles, and the alignment of 
the teeth by applying force. This type of device can be used in all age groups, but the highest efficiency was observed when is used in primary dentition in children aged three years [14]. LM TrainerTM 2 is a medical device used with beneficial effects in children aged 3 years and is effective in correcting tooth eruption. The treatment had the advantage of being effective in restoring the physiological occlusion and in eliminating the need for further treatment.

The purpose of the present study was to evaluate the toxicological profile of the biological environment of these orthodontic appliances by both in vitro and in ovo methods, previously described in the literature as useful tools for describing the safety profile of orthodontic devices [15]. Briefly, three types of saliva with acidic, neutral, and basic $\mathrm{pH}$ were prepared to observe the influence of $\mathrm{pH}$ related to the safety profile of orthodontic devices. Saliva samples were characterized: (i) from a physico-chemical point of view (microstructural changes-Scanning Electron Microscopes (SEM) analysis) and (ii) in terms of toxicological profile, in vitro techniques conducted on human keratinocytes (cell viability assessment-Alamar blue assay, gene expression-RT-PCR) and in vivo assay (angiogenic effect-hen's egg chorioallantoic method-HET-CAM).

\section{Materials and Methods}

\subsection{Reagents}

The samples were provided by national dealers: (i) Medium Myobrace for Mixed Dentition (MB), purchased from Myofunctional Research and Co., Helensvale, Australia, (www.myobrace.com, accessed on 15 October 2021) and (ii) LM Trainer ${ }^{\mathrm{TM}} 2$ Medium (LMD), purchased from LM-Instruments Oy, Parainen, Finland (www.lm-dental.com). The reagents used for the preparation of artificial saliva were: $\mathrm{CaCl}_{2} \cdot 2 \mathrm{H}_{2} \mathrm{O}$ (purity $>99.5 \%$ ) from Honeywell Fluka ${ }^{\mathrm{TM}}$ (Charlotte, NC, USA); $\mathrm{NaCl}$ (purity $>99.5 \%$ ) from Chimopar S.A (Bucuresti, Romania); $\mathrm{CO}\left(\mathrm{NH}_{2}\right)_{2}$ (purity > 99.5\%) from Sigma-Aldrich (St. Louis, $\mathrm{MO}$, USA); $\mathrm{KCl}$ (purity > 99.8\%), $\mathrm{NaOH}$ pellets (purity $>99.3 \%$ ) from Chimreactiv (Bucuresti, Romania) and $\mathrm{HCl}$ 37\% from Honeywell Fluka ${ }^{\mathrm{TM}}$ (Charlotte, NC, USA). Phosphate saline buffer (PBS), trypsin-EDTA solution, dimethyl sulfoxide (DMSO), fetal bovine serum (FBS), penicillin/streptomycin, and Resazurin reagent were purchased from Sigma-Aldrich, Merck KgaA (Darmstadt, Germany). The cell culture medium, Dulbecco's Modified Eagle Medium (DMEM-ATCC ${ }^{\circledR} 30-2002^{\mathrm{TM}}$ ), was acquired from ATCC (American Type Cell Collection, Lomianki, Poland). Trizol reagent was purchased from Thermo Fisher Scientific, Inc. (Waltham, MA, USA), Quick-RNA ${ }^{\mathrm{TM}}$ purification kit from Zymo Research, Maxima ${ }^{\circledR}$ First Strand cDNA Synthesis Kit from Fermentas, Power SYBR-Green PCR Master Mix from Thermo Fisher Scientific, Inc. (Waltham, MA, USA), primers (18S housekeeping genes, Bax, Bcl-2, and Bad) from Thermo Fisher Scientific, Inc. (Waltham, MA, USA) and Eurogentec (Seraing, Belgium).

All reagents were of an analytical grade of purity and for cell culture use.

\subsection{Preparation of the Artificial Saliva with Different $p H$ Values}

Artificial saliva solutions with different $\mathrm{pH}$ values $\left(3.072\left(\right.\right.$ at $\left.30.7^{\circ} \mathrm{C}\right), 7.139\left(\right.$ at $\left.29.6^{\circ} \mathrm{C}\right)$, and 10.005 (at $29.5^{\circ} \mathrm{C}$ )) were prepared according to the method of Alves and co-workers [16]. First, the neutral artificial saliva $(\mathrm{pH}=7.139)$ was obtained by weighing $0.40 \mathrm{mg} / \mathrm{L} \mathrm{NaCl}$; $0.40 \mathrm{mg} / \mathrm{L} \mathrm{KCl} ; 0.80 \mathrm{mg} / \mathrm{L} \mathrm{CaCl}_{2} \cdot 2 \mathrm{H}_{2} \mathrm{O}$ and $1 \mathrm{mg} / \mathrm{L} \mathrm{CO}\left(\mathrm{NH}_{2}\right)_{2}$. All the reagents were dissolved in distilled water until a clear transparent solution was obtained. In order to achieve the basic $\mathrm{pH}$, the neutral artificial saliva was treated with $10 \mathrm{~N} \mathrm{NaOH}$ aqueous solution till the $\mathrm{pH}$ meter reached 10.005 value. For acidic $\mathrm{pH}$, the initial artificial saliva was lowered to a 3.072 value by adding $\mathrm{HCl} 37 \%$ [17]. All the $\mathrm{pH}$ solutions were measured with a Thermo Scientific Eutech pH 150 portable $\mathrm{pH}$ meter with an electrode (Thermo Scientific, Waltham, MA, USA). 


\subsection{Storage Period of Samples in Artificial Saliva}

The samples of dental appliances (MB and LMD) were separately integral immersed in solutions of artificial saliva. After immersion, all samples (MB3, MB7, MB10, LMD3, LMD7, and LMD10) were kept in an incubator with an orbital shaker (ES20/60 Biosan, Riga, Latvia) at $37^{\circ} \mathrm{C}$ for 7 days, washed with distilled water, in order to remove all the rest of artificial saliva solutions, and dried. Subsequently, the samples were subjected to physico-chemical characterization and evaluation of the toxicological profile.

\subsection{Scanning Electron Microscopy Analysis}

All six samples, together with a control sample, were subjected to scanning electron microscopy (SEM) to evaluate the microstructural changes in manufactured medical-grade material (silicone) after imitating a permanent contact of the material with artificial saliva. The SEM analysis was performed by employing a Hitachi SU8230 cold field emission STEM (Chiyoda, Tokyo, Japan).

\subsection{Cell Culture}

The present study was performed using human immortalized keratinocytes, the $\mathrm{HaCaT}$ cell line, purchased from CLS Cell Lines Service GmbH (Eppelheim, Germany) as a frozen vial. The cells were cultured in their specific medium (DMEM) supplemented with $10 \%$ FCS and $1 \%$ antibiotics (100 U/mL penicillin $/ 100 \mu \mathrm{g} / \mathrm{mL}$ streptomycin) and incubated in a humidified atmosphere $\left(37^{\circ} \mathrm{C}, 5 \% \mathrm{CO}_{2}\right)$ during the experiments.

\subsection{Cell Viability Assessment}

The cell viability assessment was performed using the Alamar blue technique. In brief, the HaCaT cells were seeded in 96-well plates $\left(10^{4}\right.$ cells $/ 200 \mu \mathrm{L} /$ well $)$ and allowed to grow until the proper confluence was reached. The cells were treated with three dilutions $(1: 4,1: 2$, and 1:1) of MB and LMD saliva samples and incubated for $72 \mathrm{~h}$. At the end of the treatment, a volume of $20 \mu \mathrm{L}$ of Alamar blue (resazurin) reagent was added to each well, followed by a $3 \mathrm{~h}$ incubation at $37^{\circ} \mathrm{C}$. The absorbance values were measured at 570 and $600 \mathrm{~nm}$ using Cytation 5 (BioTek Instruments Inc., Winooski, VT, USA). The results were expressed as a percentage of viable cells (\%) and were calculated using the formula mentioned in a previous study [18].

\subsection{Gene Expression}

RT-PCR analysis was conducted in order to establish the influence of saliva test samples on gene expression. The main step of the analysis were: (i) cell growth-the $\mathrm{HaCaT}$ cells, a number of $10^{6}$ cells per well, were seeded on six-well plates and were allowed to adhere to the plate overnight; (ii) cell treatment—on day two, cells were treated with 1:1 (v:v\%) test samples:cell culture medium mixture for $24 \mathrm{~h}$; (iii) RNA isolation-total RNA was isolated using the Trizol reagent and the Quick-RNA ${ }^{\mathrm{TM}}$ purification kit; (iv) RNA transcription-total RNA was transcribed with Maxima ${ }^{\circledR}$ First Strand cDNA Synthesis Kit and (v) quantitative real-time PCR analysis-with Quant Studio 5 real-time PCR system (Thermo Fisher Scientific, Inc., Waltham, MA, USA) in the presence of Power SYBR-Green PCR Master Mix.

\subsection{Hen's Egg Test-Chorioallantoic Membrane (HET-CAM) Assay}

To evaluate the biocompatibility and potential irritant effect of MB and LMD at the capillary level, the Hen's Egg Test (HET-CAM) method was used. For this purpose, the eggs were washed, disinfected with alcohol of $70 \%$ concentration, dated, and placed in the incubator. On the third day of incubation, perforation of the hen's eggshell was performed, and a volume of approximately $7 \mathrm{~mL}$ of albumen was extracted in order to facilitate the detachment of the chorioallantoic membranes from the inner shell of the egg, thus allowing easy visualization of vascular plexus. The next day of incubation, at the top of the egg, a window was cut, which was then covered with adhesive tape, and the eggs were 
reintroduced into the incubator until the day the experiment began. The method applied for determining the irritative potential of the samples was the one described previously in the literature and readapted to the conditions of our laboratory [19]. The experiment was performed on the 10th day of incubation. Three eggs were used for each sample, at which a volume of $500 \mu \mathrm{L}$ of each sample was added. The samples tested for the irritating effect were represented by the highest concentration (1:1) previously tested in vitro. In parallel, a positive control of $1 \%$ sodium dodecyl sulfate and a negative control of water were used. The changes determined at the level of the vascular plexus were observed for $5 \mathrm{~min}$ using a stereomicroscope (Axio CAM 105 color, Zeiss), taking two sets of pictures, one before the time of application of the sample and one after $5 \mathrm{~min}$ after application. To determine the irritant potential, three major changes at the capillary level were followed, namely: hemorrhage $(H)$, vascular lysis $(L)$, and coagulation $(C)$. Finally, the analytical method for calculating the irritation score according to the literature formula was applied [20-22]:

$$
\text { IS }=5 \times \frac{301-H}{300}+7 \times \frac{301-L}{300}+9 \times \frac{301-C}{300}
$$

Depending on the value of the irritation score, the substances can be classified as presented in Table $1[21,22]$ :

Table 1. Classification of substances according to the irritation score.

\begin{tabular}{cc}
\hline Irritation Potential & Irritation Score \\
\hline Non-irritating & $0-0.9$ \\
Irritating & $1-8.9$ \\
Severe irritating & $9-21$ \\
\hline
\end{tabular}

\subsection{Statistical Evaluation}

The GraphPad Prism 9 software (GraphPad Software, San Diego, CA, USA) was used to analyze and exhibit the statistical analysis results. One-way ANOVA test was employed to establish the statistical differences of saliva samples vs. blank sample, followed by Tukey's post-test $\left({ }^{*} p<0.05,{ }^{* *} p<0.01,{ }^{* * *} p<0.001\right.$ and $\left.{ }^{* * * *} p<0.0001\right)$.

\section{Results}

\subsection{Scanning Electron Microscopy}

The SEM images of the Medium Myobrace (MB) dental appliance, before and after a week of artificial saliva immersion at different $\mathrm{pH}$ values (acidic, neutral, and basic), are shown in Figure 1, and the SEM images of LM Trainer ${ }^{\mathrm{TM}} 2$ Medium (LM) dental appliance, before and after a week of artificial saliva immersion at different $\mathrm{pH}$ values, are shown in Figure 2. The pictures are recorded at different orders of magnitude (200, 20, and $1 \mu \mathrm{m})$.

As can be seen from the figures, regardless of the $\mathrm{pH}$ value of the artificial saliva in which the samples were immersed for 7 days, none of the solutions affect the material from which the two samples are made. Considering that the artificial saliva at different $\mathrm{pH}$ values does not change the design or material of pre-orthodontic appliances, can be considered a satisfactory result, given that these pre-orthodontic appliances are designed to align the teeth into their natural position. In addition, LM Trainer ${ }^{\mathrm{TM}} 2$ is an activator and aligner for orthodontic treatment, which means that alignment, leveling, and anteroposterior correction of the teeth can be conducted at the same time without separate phases. 


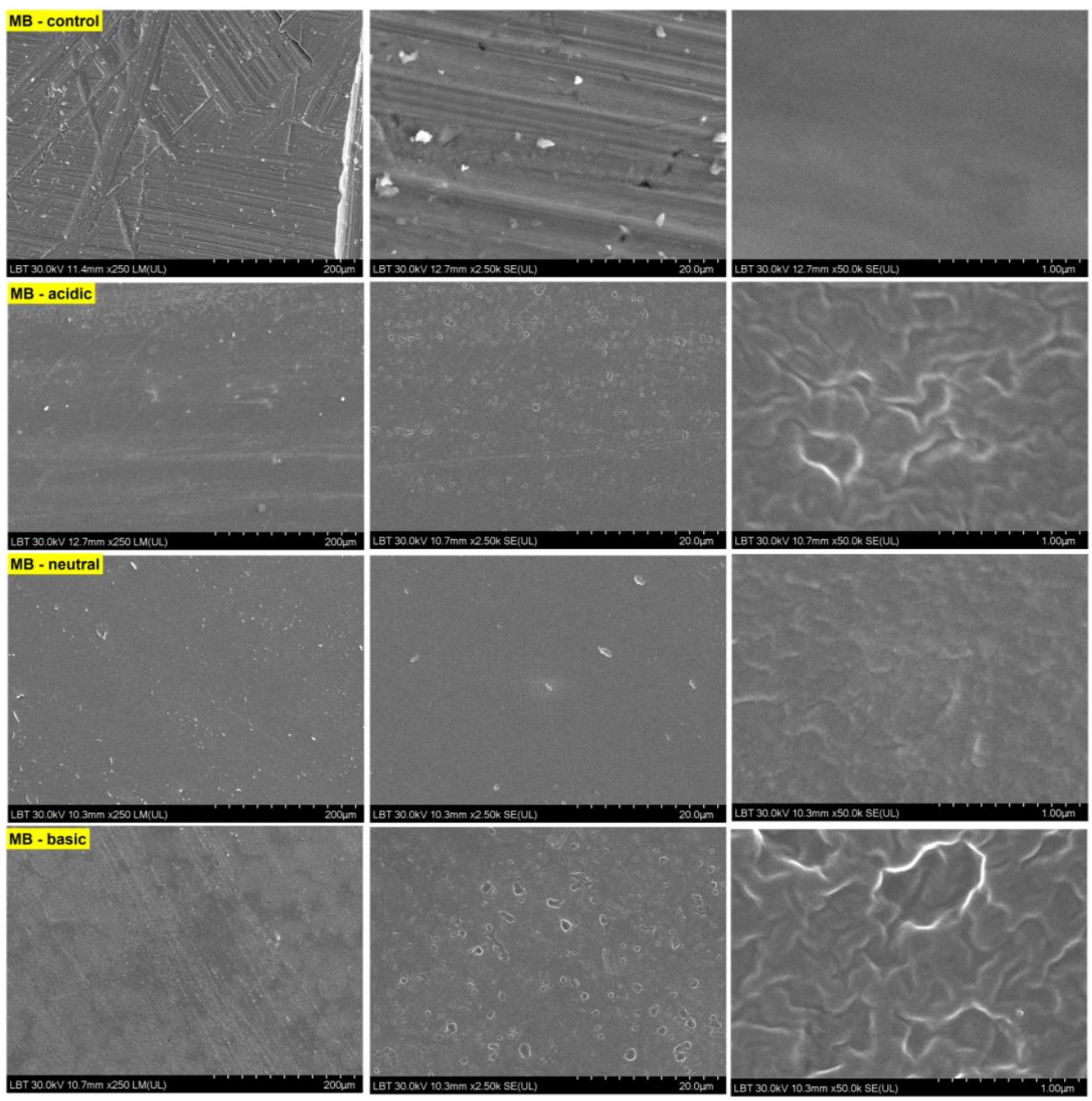

Figure 1. SEM images of Myobrance dental appliance, before (control) and after immersion, in different $\mathrm{pH}$ values of artificial saliva solutions (acidic, neutral, and basic), at various magnification orders.

\subsection{Cell Viability}

To evaluate the toxicological profile of the biological environment of MB and LMD, the saliva samples were diluted in a DMEM culture medium (1:4,1:2, and 1:1) and tested on human immortalized keratinocytes (HaCaT) for $72 \mathrm{~h}$. The viability of $\mathrm{HaCaT}$ cells was assessed by means of the Alamar blue assay at the end of a $72 \mathrm{~h}$ treatment. The results revealed slight activity of the biological environment of two materials on human keratinocytes. Thus, in the case of MB (Figure 3A), the viability percentages were almost similar to the control (untreated cells) for the diluted samples, while the lowest viability value was registered at $\mathrm{pH}=3$ for MB 1:1 (86.055\%). The cells' treatment with LMD (Figure 3B) also revealed a slight decrease in the cell viability percentages at all dilutions and $\mathrm{pH}$ values at a range between 95.095\% (MB3; 1:2) and 82.73\% (LMD3; 1:1). 


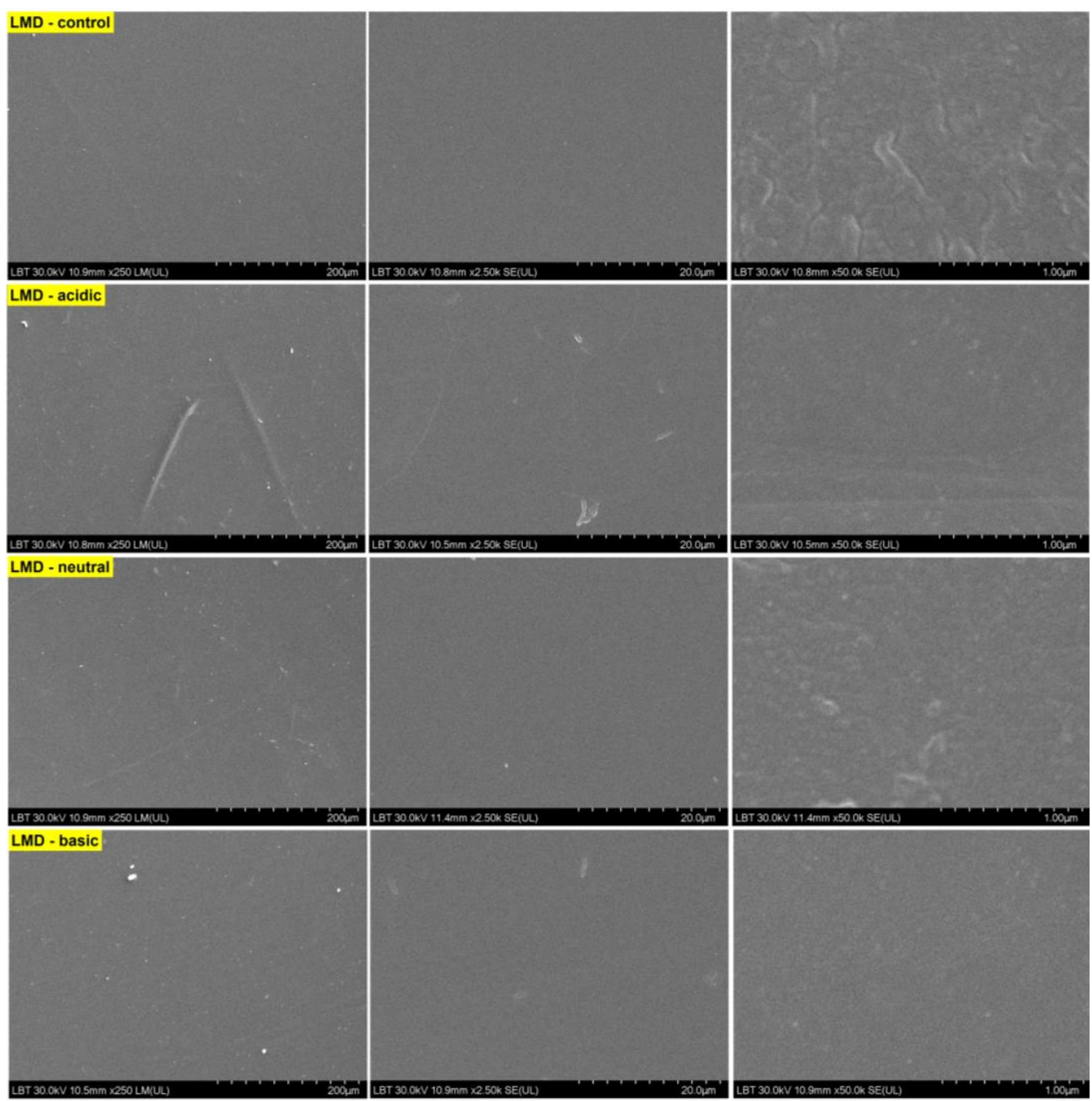

Figure 2. SEM images of LM Trainer ${ }^{\mathrm{TM}} 2$ Medium dental appliance, before (control) and after immersion, in different $\mathrm{pH}$ values of artificial saliva solutions (acidic, neutral, and basic), at various magnification orders.

A)

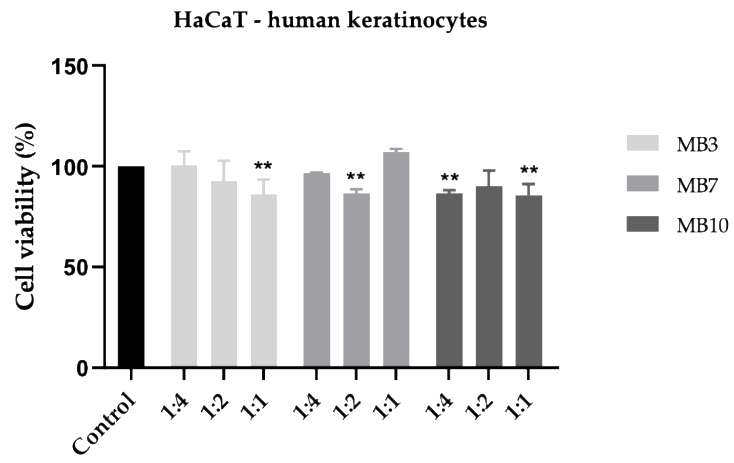

MB Dilutions
B)

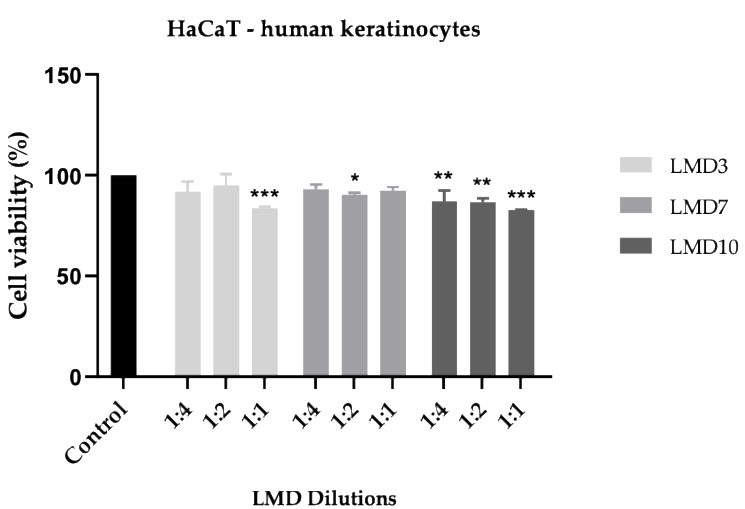

Figure 3. In vitro evaluation of the effect exerted by (A) MB and (B) $\operatorname{LMD}(1: 4,1: 2,1: 1)$ after $72 \mathrm{~h}$ of treatment on the viability of human immortalized keratinocytes (HaCaT) by performing the Alamar blue assay. Data are presented as viability percentages (\%) normalized to control and expressed as mean values $\pm \mathrm{SD}$ of three independent experiments performed in triplicate. The statistical differences between the control and the treated group were verified by applying the one-way ANOVA analysis followed by Tukey's multiple comparisons post-test $\left({ }^{*} p<0.05\right.$; ${ }^{* *} p<0.01$; $\left.{ }^{* * *} p<0.001\right)$. 


\section{3. $R T-P C R$}

Considering the results obtained from the viability test and observing that $\mathrm{MB}$ and LMD do not exert pronounced cytotoxic effects, it was decided to evaluate the main markers involved in apoptosis, namely the Bcl-2 and Bcl-xL anti-apoptotic marker and the Bad pro-apoptotic marker. The results obtained from the RT-PCR analysis performed on the human keratinocyte cell line exposed to six saliva samples showed that the samples exerted an increase in mRNA expression for the anti-apoptotic marker (Bcl-2 and Bcl-xL) (Figure 4). At the same time, a decrease in mRNA expression was found for the pro-apoptotic marker (Bad) (Figure 5).

Bcl-2

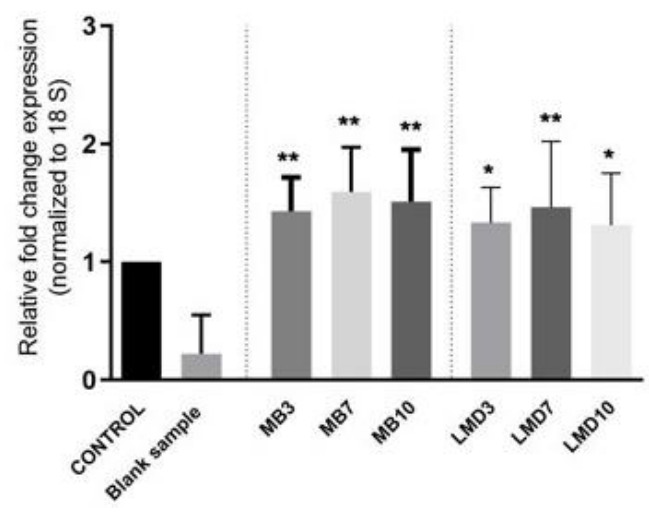

Bcl-xL

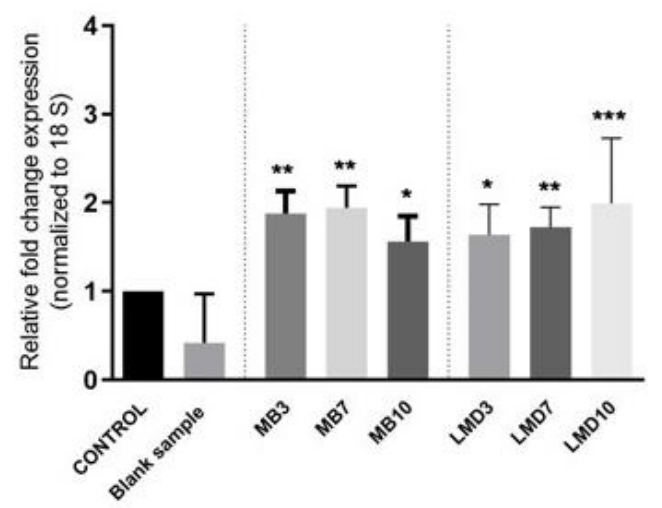

Figure 4. Relative fold change expression of mRNA of anti-apoptotic Bcl-2 and Bcl-XL markers in human immortalized keratinocytes $(\mathrm{HaCaT})-24 \mathrm{~h}$ after exposure to test samples, mRNA expression levels normalized to $18 \mathrm{~S}$ expression, mean values \pm SD of three independent experiments presented, one-way ANOVA with Tukey's post-test used to identify the statistical differences $\left({ }^{*} p<0.05,{ }^{* *} p<0.01\right.$, and $\left.{ }^{* * *} p<0.001\right)$.

\section{Bad}

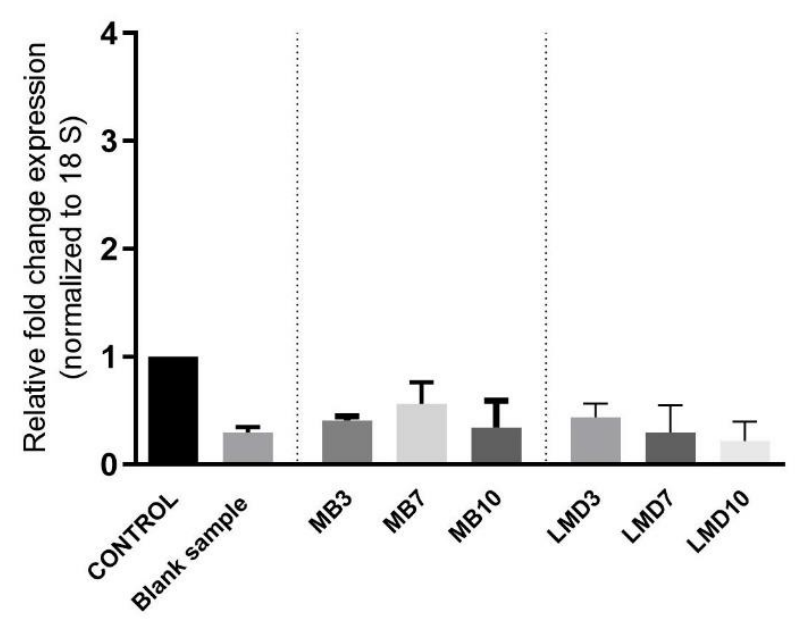

Figure 5. Relative fold change expression of mRNA of pro-apoptotic Bad marker in human immortalized keratinocytes (HaCaT) - $24 \mathrm{~h}$ after exposure to test samples, mRNA expression levels normalized to $18 \mathrm{~S}$ expression, mean values $\pm \mathrm{SD}$ of three independent experiments presented, one-way ANOVA with Tukey's post-test used to identify the statistical differences.

\subsection{MB and LMD Has No Irritative Effect in Ovo}

Toxic potential and capillary biocompatibility were analyzed using the chorioallantoic membrane by the HET-CAM test. This method allows testing the irritant effect at the application site of samples under different forms. To obtain an overview of the irritant effect and to calculate the irritation score of the samples, two controls were used, a positive 
control ( $1 \%$ sodium dodecyl sulfate), and a negative control (water). The effects observed (hemorrhage, vascular lysis, and coagulation) were recorded in the form of photographs before and after $5 \mathrm{~min}$ after application to the membrane. The values of the irritation score are presented in Table 2. It can be observed that the highest irritation score was obtained, as expected, in the case of the positive control (19.31). After applying a volume of $500 \mu \mathrm{L}$ of SDS to the vascular level, the induction of massive destructions was noted, with lysis, coagulation, and intense hemorrhage (Figure 6). In addition, the death of the specimens to which SDS was applied was recorded less than $60 \mathrm{~min}$ after application. Regarding the effect of tested samples, both MB and LMD did not exert any type of toxic change at the capillary level $(I S<0.9)$ (Figure 6). In addition, the specimens at which these samples were tested showed suitable viability, with a survival rate even after $24 \mathrm{~h}$ of application. Both the effects at the vascular level and the irritation score obtained for the tested samples were similar to those obtained for the negative control, represented by water.

Table 2. Irritation score (IS) for SDS, MB, and LMD and the occurrence time of hemorrhage (tH), lysis $(\mathrm{tL})$, and coagulation $(\mathrm{tC})$.

\begin{tabular}{ccccccccc}
\hline & SDS 1\% & $\mathbf{H}_{\mathbf{2}} \mathbf{O}$ & \multicolumn{3}{c}{ MB 1:1 } & \multicolumn{3}{c}{ LMD 1:1 } \\
\cline { 2 - 9 } & & & $\mathbf{p H ~ 3}$ & $\mathbf{p H ~ 7}$ & $\mathbf{p H ~ 1 0}$ & $\mathbf{p H ~ 3}$ & $\mathbf{p H ~ 7}$ & $\mathbf{p H ~ 1 0}$ \\
\hline $\mathbf{I S}$ & 19.31 & 0.13 & 0.82 & 0.67 & 0.73 & 0.46 & 0.28 & 0.82 \\
$\mathbf{t H}$ & $17 \mathrm{~s}$ & 300 & 300 & 300 & 300 & 300 & 300 & 300 \\
$\mathbf{t L}$ & $21 \mathrm{~s}$ & 300 & 300 & 300 & 300 & 300 & 300 & 300 \\
$\mathbf{t C}$ & $33 \mathrm{~s}$ & 298 & 275 & 280 & 278 & 287 & 293 & 275 \\
\hline
\end{tabular}
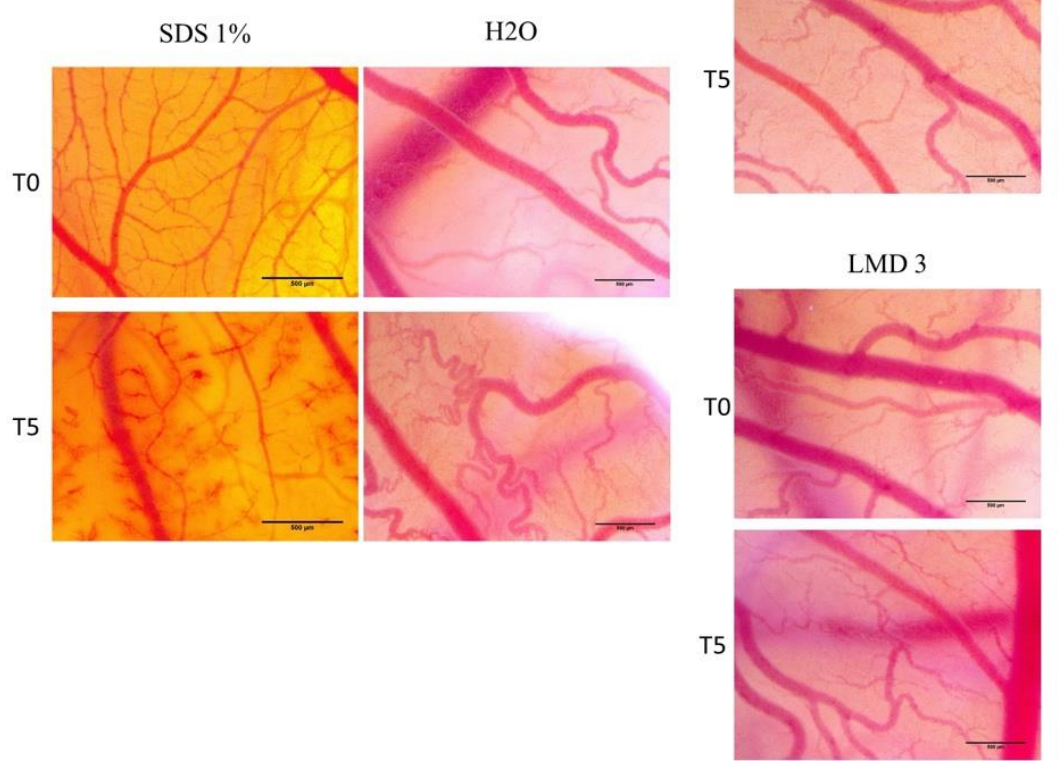
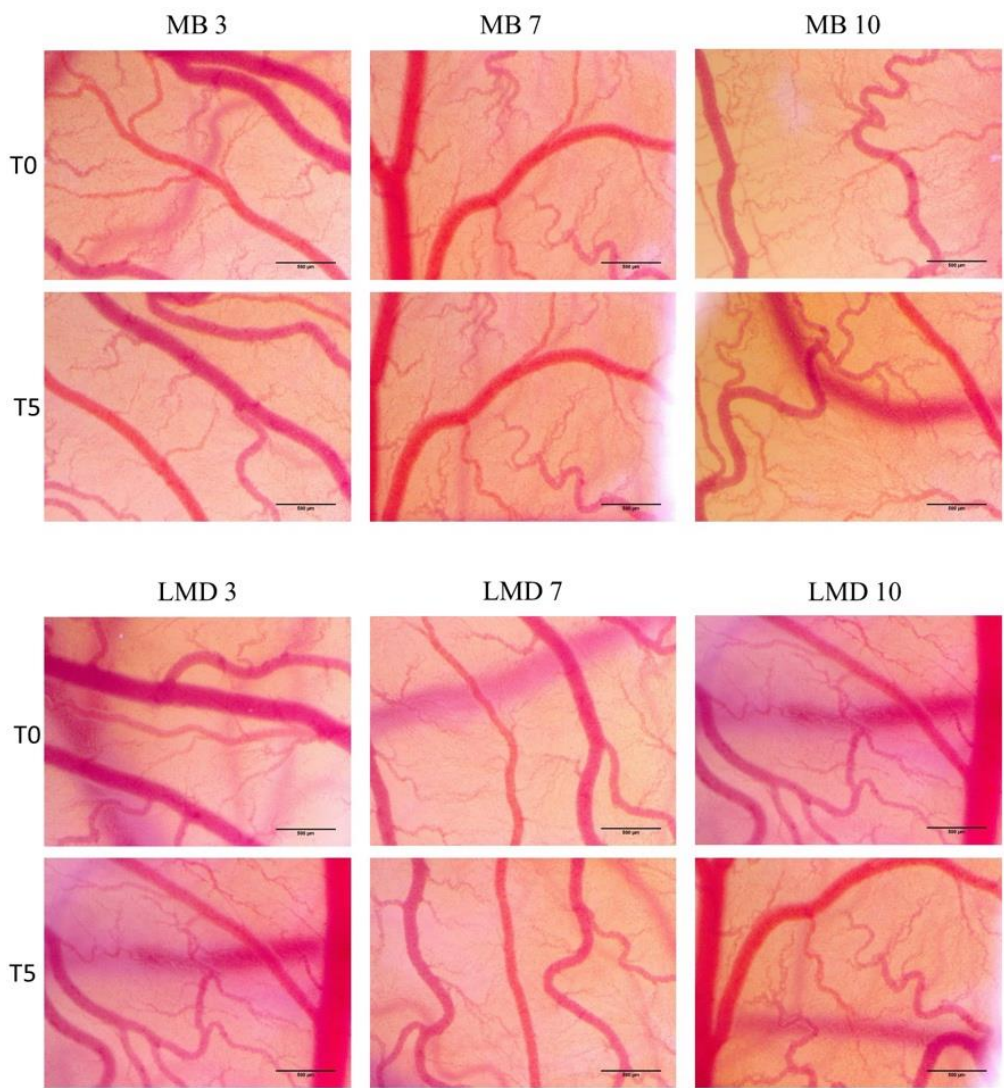

Figure 6. Analysis of the irritant potential of MB and LMD by the HET-CAM method. Stereomicroscopic images of CAMs inoculated with negative control- $\mathrm{H}_{2} \mathrm{O}$, positive control-SLS and test saliva samples. 


\section{Discussion}

The number of patients requiring orthodontic treatment due to malocclusion is constantly increasing. Discomfort caused by orthodontic appliances has a negative effect on patients' compliance with treatment [23]. Like other medical therapies, orthodontic interventions raise issues in terms of exposing patients to different types of risks. Risks associated with the use of orthodontic appliances include toothache, periodontal damage, root resorption, tooth enamel damage, and cavities [24]. Given these side effects of classical orthodontic therapy, research in the field has focused on finding new solutions. One of the elastodontic therapies for malocclusion is an alternative or myofunctional orthodontics [25]. Prefabricated myofunctional devices have gained popularity in recent years in terms of correcting various types of malocclusions in the pediatric category. The advantage of this type of therapy is that it targets the etiological factors underlying the pathology and intervenes in the correction of oropharyngeal muscle dysfunction, contributing to improving the health of the oral cavity, physical appearance, but also the posture of the tongue and airway volume. Although the effectiveness of the treatment has been proven in multiple clinical trials, the issue of compliance and safety profile remains to be discussed, even considering that this type of treatment is especially addressed to children and adolescents [26]. Orthodontic appliances are meant to stay in contact with the oral mucosa for a long time, and for this reason, biocompatibility studies are an important step in ensuring their safe use. Conventional orthodontic appliances are usually composed of metals and alloys that can exert toxic effects on the cells of the oral cavity [27]. In addition, mobile orthodontic appliances contain different metal ions that can exert toxic effects at the cellular level. Wepner and colleagues have shown that metal ions are released from orthodontic appliances depending on the $\mathrm{pH}$ of the solution [28]. They noticed that the highest concentration of metal ions released was aluminum. Similarly, Karnam and co-workers pointed out that the release of metal ions is accentuated in the first week after the devices are immersed in the solution, and then the release is slowed down [29]. In this experimental study, it was proposed to evaluate the toxicological profile of the biological environment in which two orthodontic appliances frequently used by children up to three years of age were immersed. The physico-chemical, in vitro, and in ovo methods applied in the present study for evaluation are recognized for establishing this type of toxicological profile of biological environments [30,31]. The SEM results showed no substantial morphological changes after 7 days of contact between the acidic, basic, and neutral artificial saliva and pre-orthodontic appliances. Very suitable behavior of the orthodontic appliances' materials to various acid or basic solutions is imperative because these two appliances must be worn every day and overnight while sleeping, and the degradation of the material could lead to non-conformities in its use. Thus, in this context, was assessed in vitro biocompatibility by evaluating the effect on cell viability after $72 \mathrm{~h}$ of treatment in the case of the human keratinocytes. The obtained results suggested that MB and LMD do not exert a notable cytotoxic effect, the lowest value of cell viability being obtained in the case of MB3 (dilution $1: 1$, approximately $86 \%$ ) and LMD3 (dilution 1:1, approximately $83 \%$ ). For the assessment of the in vitro toxicological profile, a very important step is to choose properly the cell line at which the tests will be performed. Therefore, the cell line sensitivity may influence the results of a cytotoxicity test. To evaluate the toxic effects of orthodontic appliances, various cell lines that mimic the environment of the oral cavity can be used. The most useful types of cell lines when testing the effects of orthodontic appliances are human keratinocytes or gingival fibroblasts [32]. In addition, the $\mathrm{HaCaT}$ cell line has the advantage of an increased proliferation rate while retaining the characteristics of the original test. For this reason, $\mathrm{HaCaT}$ is recommended for standardized testing and is widely used in scientific research [33]. In a study conducted by Locci et al. [34], the cytotoxic effects of orthodontic appliances on human gingival fibroblasts were analyzed. It has been observed that dental braces can yield metal ions such as $\mathrm{Cu}^{++}, \mathrm{Cr}^{6+}$, or $\mathrm{Ni}^{++}$. These metal ions showed cytotoxic effects in gingival fibroblasts. The strongest decrease in cell viability is observed in the case of $\mathrm{Cu}^{++}$. The alternative to conventional devices is myofunctional ther- 
apy. Myofunctional orthodontic appliances are composed of soft elastomeric material [13]. In a study realized to evaluate the cytotoxicity of elastomeric materials used in dentistry and orthodontics, it was noted that these materials do not have a cytotoxic effect on human keratinocytes (HaCaT) [35]. Thus, the cell viability recorded in this study was like that obtained in the current research. Although the myofunctional appliance is in medical use, having beneficial effects on dentition, there are no data in the literature, as far as we know, on their toxicological profile related to the biological environment. Considering the results obtained from testing on human keratinocytes, the next step of this study was to evaluate the impact of MB and LMD orthodontic appliances on the main genes involved in the apoptosis process: $\mathrm{Bcl}-2, \mathrm{Bcl}-\mathrm{xL}$, and $\mathrm{Bad}$. Data revealed that by stimulating $\mathrm{HaCaT}$ cells with artificial saliva in which MB and LMD were maintained for 7 days, there is an up-regulation of the expression of the anti-apoptotic Bcl-2 and Bcl-xL genes in parallel with a down-regulation of the expression of the pro-apoptotic gene, Bad. The apoptosis process is considered an essential marker for the evaluation of biocompatibilities and toxicity of different substances. The cellular effects exerted by a chemical can be manifested either by stimulating or depressing the mechanisms of cell death. The survival rate of cells in the presence of chemical compounds is determined by the proliferative state, the enzymatic ability to repair, and to stimulate the production of proteins capable of promoting or inhibiting the process of cell death [36]. In the case of conventional orthodontic appliances, studies have suggested that they determine the appearance of biochemical changes of the saliva as well as modification of the pathophysiological status of the entire environment of the oral cavity. Thus, regarding the process of apoptosis, it has been shown that the metal ions contained by these orthodontic treatments cause stimulation of pro-apoptotic markers and, consequently, have a cytotoxic profile for oral cells [37]. Another aspect related to conventional orthodontic therapy is the long contact time with the oral mucosa of the orthodontic appliances. Due to this long contact time, conventional braces can cause changes in DNA. A study by Kapadia et al. [38] showed that due to the content of metal ions, fixed orthodontic appliances reduce cell viability by altering DNA and promoting the process of apoptosis. Another category of orthodontic appliances is polycarbonate-based orthodontic brackets. These orthodontic appliances have also been tested for their cytotoxic effects on oral cells, and the results suggested that they have a toxic profile exerted by inducing the apoptosis process confirmed by increased levels of apoptosis inducing factor AIF protein involved in inducing apoptosis and decreased levels of Bcl-xL, an anti-apoptotic marker [39]. As previously mentioned, myofunctional orthodontic appliances are myofunctional therapy for malocclusion, being composed of soft elastomeric materials. Regarding the toxicological profile of the materials from which the two orthodontic appliances are manufactured, MB and LMD, as far as we know, no studies have been performed on their influence in the process of apoptosis. Due to the promising results observed in in vitro studies, the next direction of research was to verify the safety profile of MB and LMD in ovo at the level of the chicken egg chorioallantoic membrane. By applying the HET-CAM method, the main toxic effects exerted by a substance on the vascular plexus are followed, and finally, the calculation of an irritation score that provides an overview of the biocompatibility and toxicity of the samples [40]. The results obtained revealed that MB and LMD biological environment do not exert toxic effects at the vascular level, the irritation score obtained for all concentrations and $\mathrm{pH}$ values tested being below 0.9 and very similar to the irritation score obtained for the negative control, represented by distilled water. These results support those obtained in the case of in vitro testing and confirm the hypothesis of increased biocompatibility and lack of toxicity of the biological environment of these elastodontic dental appliances. The HET-CAM assay has been previously applied to verify the safety profile of orthodontic products as an alternative to animal testing. A study by Schendel et al. [41], which tested for 30 substances used in orthodontics, found that plastics used to make detachable devices do not produce vascular toxicity, while liquid primers for bracket bonding and monomer substances has an intense irritating effect. 


\section{Conclusions}

The elastodontic orthodontic appliances (MB and LMD) have suitable stability in the biological environment due to the materials from which they are built, giving them a biological profile suitable for clinical use. These orthodontic treatments have suitable compatibility with the biological environment. The data obtained highlight that by testing saliva in which MB and LMD were maintained, no significant decreases in cell viability were noted in the human keratinocytes and no changes in gene expression involved in the apoptosis process. Moreover, at the CAM level, no irritation changes were registered, the effects exerted by the samples being like those observed in the case of the negative control. These results support the hypothesis that MB and LMD biological environment present a safe profile and the materials have suitable stability in the biological environment, not being influenced by its $\mathrm{pH}$.

Author Contributions: Conceptualization, I.M. (Ioana Macasoi) and I.P.; Data curation, R.B., C.S.V., I.M. (Ioana Macasoi), C.A.D. and L.B.-T.; Formal analysis, R.B., M.P. (Malina Popa), I.M. (Ioana Macasoi) and L.B.-T.; Funding acquisition, M.P. (Marius Pricop), C.A.D. and E.-A.M.; Investigation, S.D., M.P., E.-A.M. and L.B.-T.; Methodology, R.B., I.M. (Ioana Macasoi), I.M. (Iasmina Marcovici), I.P., E.-A.M. and L.B.-T.; Project administration, C.A.D. and M.P. (Marius Pricop); Resources, S.D., M.P. (Malina Popa) and M.P. (Marius Pricop); Software, I.M. (Ioana Macasoi) and C.S.V.; Supervision, I.P., C.A.D. and M.P. (Marius Pricop); Validation, S.D., R.B., C.S.V. and E.-A.M.; Visualization, S.D. and C.S.V.; Writing—original draft, I.M. (Ioana Macasoi) and I.M. (Iasmina Marcovici); Writing—review and editing, I.P. and M.P. (Marius Pricop) All authors have read and agreed to the published version of the manuscript.

Funding: This research received no external funding.

Institutional Review Board Statement: Not applicable.

Informed Consent Statement: Not applicable.

Conflicts of Interest: The authors declare no conflict of interest.

\section{References}

1. Rapeepattana, S.; Thearmontree, A.; Suntornlohanakul, S. Etiology of malocclusion and dominant orthodontic problems in mixed dentition: A cross-sectional study in a group of Thai children aged 8-9 years. J. Int. Soc. Prev. Community Dent. 2019, 9, 383-399. [CrossRef] [PubMed]

2. Ahammed, A.R.; Shetty, V.; Panda, A.K.; Gunda, S.; Pradhan, D.; Husain, N.; Gugwad, S. Prevalence of malocclusion among 12 to 15 years age group orphan children using dental aesthetic index. J. Contemp. Dent. Pract. 2013, 14, 111-114. [CrossRef]

3. Anthony, S.N.; Zimba, K.; Subramanian, B. Impact of Malocclusions on the Oral Health-Related Quality of Life of Early Adolescents in Ndola, Zambia. Int. J. Dent. 2018, 2018, 7920973. [CrossRef]

4. Dutra, S.R.; Pretti, H.; Martins, M.T.; Bendo, C.B.; Vale, M.P. Impact of malocclusion on the quality of life of children aged 8 to 10 years. Dent. Press J. Orthod. 2018, 23, 46-53. [CrossRef]

5. Mageet, A.O. Classification of Skeletal and Dental Malocclusion: Revisited. Stomatol. Edu. J. 2016, 3, 205-211. [CrossRef]

6. Yadav, D.; Rani, M.; Shailaja, A.; Anand, D.; Sood, N.; Gothi, R. Angle's Molar Classification Revisited. J. Indian Orthod. Soc. 2014, 48, 382-387. [CrossRef]

7. Buschang, P.H. Class I malocclusions-The development and etiology of mandibular malalignments. Semin. Orthod. 2014, 20, 3-15. [CrossRef]

8. Eliades, T.; Hegdvedt, A.K. Orthodontic-surgical correction of a Class II, Division 2 malocclusion. Am. J. Orthod. Dentofac. Orthop. 1996, 110, 351-357. [CrossRef]

9. Li, C.; Cai, Y.; Chen, S.; Chen, F. Classification and characterization of class III malocclusion in Chinese individuals. Head Face Med. 2016, 12, 1-8. [CrossRef] [PubMed]

10. American Academy on Pediatric Dentistry Clinical Affairs Committee-Developing Dentition Subcommittee. American Academy on Pediatric Dentistry Council on Clinical Affairs. Management of the Developing Dentition and Occlusion in Pediatric Dentistry. Pediatr. Dent. 2017, 39, 334-347.

11. Saccomanno, S.; Antonini, G.; D'Alatri, L.; D'Angelantonio, M.; Fiorita, A.; Deli, R. Patients treated with orthodon-ticmyofunctional therapeutic protocol. Eur. J. Paediatr. Dent. 2012, 13, 241-243. [PubMed]

12. Melchior, M.d.O.; Magri, L.V.; Mazzetto, M.O. Orofacial myofunctional disorder, a possible complicating factor in the management of painful temporomandibular disorder. Case report. Braz. J. Pain 2018, 1, 80-86. [CrossRef] 
13. Wishney, M.; Darendeliler, M.A.; Dalci, O. Myofunctional therapy and prefabricated functional appliances: An overview of the history and evidence. Aust. Dent. J. 2019, 64, 135-144. [CrossRef] [PubMed]

14. da Cunha Busquet, P.; de Jesus Portelinha, D.; da Costa, M.L.; de Andrade Cancio de Paula, V. How the myobrace appliance works: Advantages and disadvantages. J. Dent. Probl. Solut. 2021, 8, 19-23. [CrossRef]

15. Galeotti, A.; Uomo, R.; Spagnuolo, G.; Paduano, S.; Cimino, R.; Valletta, R.; D'Anto, V. Effect of pH on in vitro biocompatibil-ity of orthodontic miniscrew implants. Prog. Orthod. 2013, 14, 1-7. [CrossRef]

16. Alves, C.B.C.; Segurado, M.N.; Dorta, M.C.L.; Dias, F.R.; Lenza, M.G.; Lenza, M.A. Evaluation of cytotoxicity and corrosion re-sistance of orthodontic mini-implants. Dent. Press J. Orthod. 2016, 21, 39-46. [CrossRef]

17. Geramipanah, F.; Majidpour, M.; Sadighpour, L.; Fard, M.J.K. Effect of artificial saliva and pH on shear bond strength of resin cements to zirconia-based ceramic. Eur. J. Prosthodont. Restor. Dent. 2013, 21, 5-8.

18. Maghiari, A.L.; Coricovac, D.; Pinzaru, I.A.; Macașoi, I.G.; Marcovici, I.; Simu, S.; Navolan, D.; Dehelean, C. High Concentra-tions of Aspartame Induce Pro-Angiogenic Effects in Ovo and Cytotoxic Effects in HT-29 Human Colorectal Carcinoma Cells. Nutrients 2020, 12, 3600. [CrossRef]

19. Macașoi, I.; Pavel, I.Z.; Moacă, A.E.; Avram, Ș.; David, V.L.; Coricovac, D.; Mioc, A.; Spandidos, D.A.; Tsatsakis, A.; Soica, C.; et al. Mechanistic investigations of antitumor activity of a Rhodamine B-oleanolic acid derivative bioconjugate. Oncol. Rep. 2020, 44, 1169-1183. [CrossRef]

20. Batista-Duharte, A.; Jorge Murillo, G.; Pérez, U.M.; Tur, E.N.; Portuondo, D.F.; Martínez, B.T.; Téllez-Martínez, D.; Betancourt, J.E.; Pérez, O. The Hen's Egg Test on Chorioallantoic Membrane: An Alternative Assay for the Assessment of the Irritating Ef-fect of Vaccine Adjuvants. Int. J. Toxicol. 2016, 35, 627-633. [CrossRef]

21. Budai, P.; Kormos, É.; Buda, I.; Somody, G.; Lehel, J. Comparative evaluation of HET-CAM and ICE methods for objective as-sessment of ocular irritation caused by selected pesticide products. Toxicol. Vitr. 2021, 74, 105150. [CrossRef] [PubMed]

22. Guran, K.; Buzatu, R.; Pinzaru, I.; Boruga, M.; Marcovici, I.; Coricovac, D.; Avram, S.; Poenaru, M.; Susan, M.; Susan, R.; et al. In Vitro Pharmaco-Toxicological Characterization of Melissa officinalis Total Extract Using Oral, Pharynx and Colorectal Carcinoma Cell Lines. Processes 2021, 9, 850. [CrossRef]

23. Chen, J.; Wan, J.; You, L. Speech and orthodontic appliances: A systematic literature review. Eur. J. Orthod. 2018, 40, 29-36. [CrossRef] [PubMed]

24. Wishney, M. Potential risks of orthodontic therapy: A critical review and conceptual framework. Aust. Dent. J. 2017, 62, 86-96. [CrossRef] [PubMed]

25. Gökçe, B. Current Approaches in Myofunctional Orthodontics. J. Musculoskelet. Disord. Treat. 2016, 2, 022. [CrossRef]

26. Elhamouly, Y.; El-Housseiny, A.A.; Ismail, H.A.; El Habashy, L.M. Myofunctional Trainer versus Twin Block in Developing Class II Division I Malocclusion: A Randomized Comparative Clinical Trial. Dent. J. 2020, 8, 44. [CrossRef]

27. Olszewska, A.; Hanć, A.; Barałkiewicz, D.; Rzymski, P. Metals and Metalloids Release from Orthodontic Elastomeric and Stainless Steel Ligatures: In Vitro Risk Assessment of Human Exposure. Biol. Trace Elem. Res. 2020, 196, 646-653. [CrossRef]

28. Wepner, L.; Färber, H.A.; Jaensch, A.; Weber, A.; Heuser, F.; Keilig, L.; Singer, L.; Bourauel, C.P. In vitro ion release of wires in removable orthodontic appliances. Materials 2021, 14, 3402. [CrossRef]

29. Karnam, S.K.; Reddy, A.N.; Manjith, C.M. Comparison of metal ion release from different bracket archwire combinations: An in vitro study. J. Contemp. Dent. Pract. 2012, 13, 376-381.

30. Szuhanek, C.A.; Watz, C.G.; Avram, S.; Moacă, E.-A.; Mihali, C.V.; Popa, A.; Campan, A.A.; Nicolov, M.; Dehelean, C.A. Comparative Toxicological In Vitro and In Ovo Screening of Different Orthodontic Implants Currently Used in Dentistry. Materials 2020, 13, 5690. [CrossRef]

31. Popa, A.; Dehelean, C.; Calniceanu, H.; Watz, C.; Brad, S.; Sinescu, C.; Marcu, O.A.; Popa, C.S.; Avram, S.; Nicolov, M.; et al. A Custom-Made Orthodontic Mini-Implant-Effect of Insertion Angle and Cortical Bone Thickness on Stress Distribution with a Com-plex In Vitro and In Vivo Biosafety Profile. Materials 2020, 13, 4789. [CrossRef] [PubMed]

32. Martín-Cameán, A.; Jos, Á.; Mellado-García, P.; Iglesias-Linares, A.; Solano, E.; Cameán, A.M. In vitro and in vivo evidence of the cytotoxic and genotoxic effects of metal ions released by orthodontic appliances: A review. Environ. Toxicol. Pharmacol. 2015, 40, 86-113. [CrossRef] [PubMed]

33. Wataha, J.C.; Hanks, C.T.; Sun, Z. Effect of cell line on in vitro metal ion cytotoxicity. Dent. Mater. 1994, 10, 156-161. [CrossRef]

34. Locci, P.; Lilli, C.; Marinucci, L.; Calvitti, M.; Belcastro, S.; Bellocchio, S.; Staffolani, N.; Guerra, M.; Beccheti, E. In vitro cyto-toxic effects of orthodontic appliances. J. Biomed. Mater. Res. 2000, 53, 560-567. [CrossRef]

35. Goiato, M.; Nobrega, A.; Malavazi, E.; Takamiya, A.; Penha de Oliveira, S. In Vitro Analysis of the Proliferation of HaCaT Cells Stimulated by Pigments Used for Maxillofacial Prostheses. J. Orofac. Sci. 2019, 11, 32-36. [CrossRef]

36. Orrenius, S.; Nicotera, P.; Zhivotovsky, B. Cell Death Mechanisms and Their Implications in Toxicology. Toxicol. Sci. 2011, 119, 3-19. [CrossRef]

37. Buczko, P.; Szarmach, I.; Grycz, M.; Kasacka, I. Caspase-3 as an important factor in the early cytotoxic effect of nickel on oral mucosa cells in patients treated orthodontically. Folia Histochem. Cytobiol. 2017, 55, 37-42. [CrossRef]

38. Kapadia, J.M.H.; Agarwal, A.R.; Mishra, S.; Joneja, P.; Yusuf, A.S.; Choudhary, D.S. Cytotoxic and genotoxic effect on the buc-cal mucosa cells of patients undergoing fixed orthodontic treatment. J. Contemp. Dent. Pract. 2018, 19, 1358-1362. [CrossRef] 
39. Kloukos, D.; Taoufik, E.; Eliades, T.; Katsaros, C.; Eliades, G. Cytotoxic effects of polycarbonate-based orthodontic brackets by activation of mitochondrial apoptotic mechanisms. Dent. Mater. 2013, 29, e35-e44. [CrossRef]

40. Mioc, M.; Avram, S.; Bercean, V.; Porcarasu, M.B.; Soica, C.; Susan, R.; Kurunczi, L. Synthesis, Characterization and Antiproliferative Activity Assessment of a Novel 1H-5-mercapto-1,2,4 Triazole Derivative. Rev. Chim. 2017, 68, 745-747. [CrossRef]

41. Schendel, K.U.; Erdinger, L.; Komposch, G.; Sonntag, H.G. Orthodontic materials studied in the HET-CAM test for muco-sairritating effects. Fortschr. Kieferorthop. 1994, 55, 28-35. [CrossRef] [PubMed] 